\title{
APURDUTM
}

Euskal ikerketen aldizkaria | Revue d'études basques |

Revista de estudios vascos | Basque studies review

2| 1997

Numéro II

\section{L'identité basque dans l'œuvre de M. de Larramendi, S.J. (1690-1766)}

\section{Txomin Peillen}

\section{OpenEdition}

\section{Journals}

Édition électronique

URL : http://journals.openedition.org/lapurdum/1806

DOI : 10.4000/lapurdum.1806

ISSN : 1965-0655

\section{Éditeur}

IKER

Édition imprimée

Date de publication : 1 octobre 1997

Pagination : 121-128

ISBN : 2-84127-142-0

ISSN : $1273-3830$

Référence électronique

Txomin Peillen, «L'identité basque dans l'œuvre de M. de Larramendi, S.J. (1690-1766) », Lapurdum [En ligne], 2 | 1997, mis en ligne le 01 septembre 2010, consulté le 29 janvier 2020. URL : http:// journals.openedition.org/lapurdum/1806 ; DOI : 10.4000/lapurdum.1806 
Txomin PEILLEN

\section{L'IDENTITÉ BASQUE DANS L'GUVRE DE M. DE LARRAMENDI, S.J. (1690-1766)}

Le Siècle des Lumières valut au Pays Basque l'apparition de plusieurs réflexions sur les identités culturelle et nationale du peuple basque, en contradiction avec le libéralisme et le césarisme politique de l'llustración espagnole. Dans l'œuvre du Père Larramendi, connue dans toute l'Espagne et une partie de l'Europe, nous distinguerons deux sortes d'ouvrages, ceux destinés à l'Espagne et ceux destinés aux Basques.

Le document de base dans le premier cas sera la préface du Diccionario Trilingue de 1745 où les Basques sont définis à l'intérieur de l'hispanité. Le second type d'ouvrage est son recueil Sobre los Fueros de Guipuzcoa, (1745-1765) ensemble de conférences prononcées en public, devant les Batzar Orokor ou Juntas Generales de Guipuzcoa. Après son séjour à Bayonne (1730-1733) il définit l'identité basque contre cette même hispanité. Il n'est pas douteux que ces textes furent connus des frères Garat qui demandèrent un département basque à la Convention, et firent à Napoléon $\mathrm{I}^{\text {er }}$ des propositions identiques à celles de Manuel de Larramendi, d'un royaume basque des sept provinces. Les conférences de Larramendi, vu leur virulence, ne purent être publiées qu'au XX' siècle

Des historiens du Pays Basque ne reconnaissent pas au Père Larramendi la paternité du nationalisme pourtant clairement exprimé sous le prétexte qu'il n'aurait pas fondé de mouvement ni de parti nationaliste. Soyons sérieux ! Larramendi est du $\mathrm{XVIII}^{\mathrm{e}}$ siècle et la question des nationalités ne se posa en Europe que cent ans après. De même les partis politiques au sens moderne du terme n'apparaîtront qu'au siècle suivant. Notre but sera de démontrer textes à l'appui l'apport de notre jésuite à la réflexion sur l'identité basque et les relations avec des pensées anciennes plus confuses et des mythes historiques hérités des XV' et XVI" siecles.

L'ensemble des textes de Sobre los fueros de Guipuzcoa. comprend trois parties et contient des allusions aux autres provinces basques. Il est d'ailleurs troublant de trouver vingt ans plus tard, en basque, des textes d'un enseignant souletin du collège de Mauléon qui répètent l'argumentation de Larramendi qu'il cite sans cesse, quant à l'identité culturelle (les douze qualités de la langue basque) et dans sa défense des fors souletins.

Les trois parties du texte de Larramendi sont une étude de l'origine des fueros guipuzcoans, une étude de l'avenir de ceux-ci et une étonnante et première définition complète de la revendication protonationaliste basque.

\section{L'origine des fors et les Basques hommes toujours libres}

Dès la première conférence nous lisons sur l'ancienneté du droit basque que

"Nous voyons le Guipuzcoa vivre depuis toujours et sans interruption avec les activités et les conséquences bénéfiques des Fors et que cela est connu et lui est reconnu par l"Espagne entière" (op. cit. page 10, traduction Tx. P.) 
Pour préciser la nature des fors il rappelle que :

"Les fors n'ont pas été écrits sous le titre d'Ordonnances ou de Privilèges, mais approuvés par le Roi et le Tribunal Suprème de Castille" (op. cit. p. 11)

Puis il nous parle de la liberté originelle des Basques, de celle qu'ils ont connue avant les monarchies, de celle qu'ils connaissent depuis qu'ils sont retirés et repoussés dans leurs "montagnes- citadelles" :

"Pero retirado a estos Montes Cantabros como a ciudadela inexpugnable"

(Prologue du Dictionnaire Trilingue de 1755).

Il insiste sur le fait qu'avant que n'existe un roi ou que les Basques en aient un, l'on n'a jamais écrit ou dit que les Basques n'étaient pas des hommes libres sur leur terre. Page 16 de son ouvrage sur les fueros il rappelle la formule de serment exigé du roi d'Espagne pour que les Basques l'acceptent comme roi :

"He venido en declarar y mandar en su consecuencia que a la provincia de Guipúzcoa se mantenga en sus Fueros, privilegios y costumbres como los ha gozado y debido gozar hasta ahora".

Larramendi distingue bien les deux adversaires de ces fors, d'une part les juristes castillans qui expriment la pensée absolutiste des Bourbons nouvellement montés sur le trône d'Espagne, d'autre part les riches libéraux partisans de l'uniformisation de ]'Espagne qui favoriserait leur développement capitaliste. Plus loin il reconnaîtra que la seconde catégorie n'est pas rare parmi les Basques, bradeurs de leur liberté, parfois en échange de quelques repas annuels.

\section{De la nécessité des Fors pour l'avenir de l'identité basque}

\section{Les menaces de l'époque contre les Fueros.}

Dans tous ses livres Larramendi définit le Basque, à l'instar de tous nos historiens basques des XVIc et XVII ${ }^{e}$ siècles, comme un homme libre, comme un homme noble, comme un homme qui a choisi son roi. Aussi la première étape de son projet politique est la consolidation prioritaire des Fueros et leur défense. Il résume et renouvelle l’argumentation des origines:

“le droit guipuzcoan ne se discute pas, alors qu' on veut nous le nier, il n'est pas comparable à celui de Castille, il n'est pas une fantaisie, un caprice, il n'est pas récent, il n'est pas d'hier, mais très ancien ; on n'en trouvera pas le début, puisque nous l'avions bien avant de nous unir à la Castille et même avant l'union avec la Navarre (op. cit. p. 97).

Les historiens et juristes espagnols centralistes de l'époque tentaient déjà d'entretenir une confusion entre le fuero de la ville, charte de fondation accordée par le roi et le Fuero General, ensemble de lois reconnu et juré par le roi.

Le Guipuzcoa ne cédera pas sur ses Fors et ses libertés.

En premier le Gujpuzcoa ne peut écarter le service de Dieu, pour celui du Roi.

En second, parce qu'il perdrait sa gloire, son renom, son honneur et sa noblesse.

En troisième lieu parce que ce serait, les ayant reçus des premiers habitants, une perte pour nos descendants. (op. cit. p.130-135) 
La conclusion est ferme :

"On ne peut laisser au pouvoir du Roi la possibilité de prêter ou de ne pas prêter serment." (op. cit. p.139)

Et les conséquences sont poussées à l'extrème :

"il y a d'autres façons d'user de la violence pour rendre nuls les contrats et ce sont ceux qui se pratiquent en Guipuzcoa "(op. cit. p.165)

Deux voies sont proposées pour lutter contre la tyrannie royale des nouveaux rois Bourbons le "pase foral" où on obéit aux ordres du Roi, sans les exécuter."

"Le roi qui pratique injustice et "anti-forisme "ne mérite plus le titre de Roi mais reste avec celui méprisable de tyran, et alors il ne faudra pas suivre ses ordres et ses décisions, ce que l'on appelle obéir et ne pas exécuter (op. cit.p.197)

L'autre voie conduit à la révolte armée contre le tyran :

"Se contiene la conclusión presente de que pueden defenderse por armas cuando un Rey quiere atropellarlos tiránicamente." (op. cit. p.197).

Il n'est pas étonnant de lire dans "Mosde Etxeberriren gogamenak" du souletin J. Egiategi vers 1780 les mêmes considérations sur la tyrannie royale française : l'idée était commune aux penseurs de ce siècle.

\section{Des mauvaises influences françaises}

Parmi les menaces françaises il analysera plus loin le comportement du centralisme royal contre les fors du Pays Basque nord, mais pour le Guipuzcoa il craint deux choses, le jansénisme (puisqu'il est jésuite) et le principe de la royauté de droit divin qui va à l'encontre des idées ibériques sur la question et du projet de Larramendi que nous analyserons plus loin.

Scandalisé par la résistance des jansénistes (dont trois bayonnais) à Louis XIV il la qualifie de "rebelión sostenida de los maniáticos" et les même maniaques révoltés sont qualifiés de "nacion hypócrita de los jansenistas. "Larramendi craint plus les jansénistes que les protestants puisque la vision des premiers est opposée à celle des jésuites. Ayant séjourné trois ans à Bayonne il n’était pas sans connaître les Messieurs de Camp de Prats, Jansen et compagnie. Il accuse d'ailleurs le gallicanisme d'avoir servi de couverture au jansénisme.

"Les libertés de l'Eglise de France que nous voyons ramper, servent de couverture et de prétexte aux Jansénistes et permettent de cacher des hérésies, des schismes des révoltes tant de fois condamnées" (op. cit. p.204).

Voici son argumentation, sur la Royauté :

"Contrairement à ce que certains croient (les Français et les Bourbons) vous devriez savoir que Je pouvoir du Roi ne vient pas directement de Dieu mais indirectement, parce que la société et les peuples ont été créés par Dieu".

\section{(op. cit. p.226)}

Dans l'esprit de Larramendi la souveraineté est donc populaire, elle appartient au pays et non au Roi : cette argumentation n'est pas inconnue des juristes hollandais, espagnols et même français, de l'époque et depuis le XVI' siècle, pour le moins.

\section{Le combat pour les Fueros}

Larramendi prophète ? On pourrait le penser quant il écrit : 
"Si le Guipuzcoa refuse d'exécuter les ordres, des décisions pourront lui ôter ses franchises, et en placer la frontière au bord de la mer." (op. cit.178)

En effet c'est à cette époque que Philippe V tentera de supprimer les ports francs basques et de ramener les douanes de I'Ebre au bord des rivages cantabriques. Il y renoncera par suite de l'opposition sanglante de tous les Basques, puis de la guerre contre la France, mais après la seconde guerre carliste en 1877 le Pays Basque sud perdra son autonomie et ne sera plus une zone franche.

D'ailleurs l'intuition et la prophétie larramendiennes vont plus loin, puisqu'il pense que pour défendre ses libertés contre l'Espagne, le Guipuzcoa ne pourra pas aller au combat seule, et la prémonition des guerres carlistes est claire :

"Et si le Guipuzcoa n'est pas avec ses habitants suffisant à riposter aux ennemis, nous pourrions appeler nos concitoyens amis de France, de Navarre, d'Alava, de Biscaye et le Guipuzcoa se donner à un autre Roi qui protégerait ses fors et ses libertés”. (op. cit. p.282)

Larramendi imagine ces guerres forales, même s'il pense que l'on n'en arrivera pas à ce point là. Ces combats, s ils ont lieu, seront rudes mais pas inorganisés : il devront être contrôlés, commandés par les Juntas Generales.

"Pour lutter contre les injustices et les violences faites aux Fueros, il ne faut pas accepter des désordres populaires, au contraire les actions devront être menées après décision des Juntas Generales, en ordre, en bonne entente”. (op. cit. p. 282)

\section{La dernière étape : la République des Provinces Unies des Pyrénées}

Informé des théories et de l'organisation qui permirent aux Pays Bas de se séparer de l'Espagne et ayant à l'esprit les expériences des Missions jésuites des pays guaranis, Larramendi va plus loin et propose de fonder entre les sept provinces basques une nation indépendante avec une royauté élective, altemant entre toutes les provinces basques. Le roi jurerait une constitution ou Pacta Conventa et serait un militaire

Devant les graves menaces qui pèsent sur les Fueros du sud et les Fors du nord du Pays Basque du fait des Bourbons centralisateurs, Larramendi propose plusieurs solutions qui sont toutes des ruptures avec l'Espagne :

“La seigneurie de Biscaye comme nous commence à gémir sous le joug. Aux uns et autres, demandons-leur de prendre le nom de Provinces Unies des Pyrénées".

La principale argumentation repose sur l'identité culturelle :

"Pour quoi la langue basque aussi vive et plus vivace que beaucoup d'autres ne doit -elle pas voir tous ses bascophones réunis dans une seule nation? Pour quoi les trois provinces d'Espagne (Guipuzcoa, Alava, Biscaye) et le royaume de Navarre doiventils être dépendants de la Castille et le Labourd, la Soule, la Basse-Navarre dépendre de la France? (op. cit. p.58)

\section{Trois solutions sont proposées :}

La première une nation sous protection anglaise, la seconde possibilité une nation sous protection française, la troisième solution une nation totalement indépendante : il est donc le père du nationalisme idéologique basque

Avec l'Angleterre. Sous cette protection le commerce basque serait plus brillant, le Guipuzcoa vendrait mieux son acier et les nombreux produits anglais parviendraient à meilleur prix. Cela permettrait aussi politiquement d'équilibrer l'Europe, face 
au bloc Espagne-France des Bourbons. (op. cit. p.56). Bien que le Royaume Uni soit protestant et en réservant les droits des catholiques basques, l'entente serait possible avec la couronne anglaise.

Avec la France. Tout est problématique, malgré le voisinage, parce que la monarchie française ne tarderait pas à supprimer les Fueros et les Juntas generales, comme elle a commencé à le pratiquer dans ses pays d'Etats. Par ailleurs Larramendi craint la contamination janséniste, qu'il a bien connue à Bayonne lors de son séjour dans cette ville. Cependant cette solution permettrait d'éviter les guerres entre la France et l'Espagne. et l'idée de l'état-tampon que développera Joseph Garat auprès de Napoléon apparaît ici.

\section{L'organisation de la "république" indépendante.}

Larramendi préfere la troisième solution, même si elle lui semble la plus ardue, la plus longue à mener et à maintenir.

“Nous créerons entre tous les Basques une république.....Si cela nous convient nous choisirons un Roi et pour notre profit ce roi sera élu. Nous le choisirons à tour de rôle dans toutes nos provinces. Ce roi devrait avant tout recevoir une formation militaire, être d'un grand courage, d'une énergie farouche pour mener à bien et faire durer notre action héroïque" (op. cit. p. 59).

L'élection du roi qui revient dans la pensée de cet homme du XVIII' siècle n'est pas chose nouvelle, les Francs à l'origine, puis les Castillans, les Navarrais, les Aragonais, la pratiquèrent longtemps et les "dynasties" furent parfois courtes. La nouveauté est la nécessité d'une sorte de général à la tête de la république, mais cela s'explique par les propos de notre auteur dans les pages 67 et suivantes.

Le militaire. Le Pays Basque pris entre deux voisins puissants, qui l'ont toujours convoité, devra pour durer être organisé militairement : une armée de 30.000 hommes et tous les autres armés à domicile, (comme les Helvètes). Il faudra prévoir dans tout les pays des dépôts d'armes et munitions discrets. La marine de guerre devra être importante.

Le civil. Les Fueros devront veiller à deux écueils : interdire la jurisprudence justinienne cause de la ruine des gens en procès, et d'autre part combattre le luxe source également de dégâts économiques pour les petits états (et obsession des jésuites depuis leur origine). La même argumentation est soutenue par le souletin J. Egiategi en basque, dans son livre de philosophie de 1785 "Filosofo heiiskaldiunaren ekheia". Les lois seront modifiées par les Basques sans intervention de quelque roi que ce soit. La langue des institutions politiques et des assemblées sera le basque : ceux qui ne le savent pas l'apprendront, comme les Basques l'ont fait avec le castillan.

L'Education. Dans cette république la petite école enseignera uniquement le basque et le latin : Dans le secondaire tout le monde apprendra quatre langues : basque, latin, français, espagnol, puis les sciences des mathématiques, de l'agronomie, de la navigation. Larramendi avoue que jusque-là et comme la Castille respectait leurs Fueros, par loyauté, les Basques du sud ont choisi d'écrire en castillan, mais que devant la dégradation politique des relations evec Madrid, de la baisse de qualité de la langue, $c^{\prime}$ est le basque qu'il faut porter à l'école.

La constitution. Le roi devra prêter serment en plus des Fueros à une Constitution ou Pacta Conventa. Cette république ne sera viablẹ que si au Guipuzcoa s'ajoutent les six autres provinces basques. 
Quand et comment? Larramendi pense que la république des Provinces Unies des Pyrénées sera Iongue à constituer, face au harcèlement castillan et à l'inquiétante évolution préjacobine de la France. Il est intéressant de remarquer que ce projet opposé à celui des "Lumières" espagnoles n'est pas césarien comme le leur, et qu'il est plus moderne que celui de Sabino Arana Goiri qui au départ plongea ses racines dans les obscurités du XIX ${ }^{c}$ siècle et les débuts du capitalisme sauvage en Biscaye.

Les conceptions semi-mythiques de lidentité culturelle basque chez Larramendi passèrent au Pays Basque nord où sa grammaire et son dictionnaire trilingue furent connus. Peut-être furent-ils apportés par l'auteur au cours de séjours à Bayonne et par des contacts que les gens du Pays Basque nord maintinrent avec Loiola où l'auteur s'était retiré.

Ses écrits politiques n'eurent pas la même diffusion au nord mais il est certain que l'idée des Guipuzcoans de s'unir à la France au tout début de la Révolution, l'accueil qu'ils firent aux premières troupes s'expliquent autant par les conflits avec Madrid que par les idées semée par Larramendi et ses suiveurs.

Ce qui reste à étudier, ce sont les éléments larramendiens dans l'cuvre de Dominique Joseph Garat notamment dan son traite Recherche sur le Peuple Primitif de l'Espagne, sur les révolutions de cette péninsule, sur les basques espagnols et français (Paris 18I8) dont le titre rappelle le livre de Poza au XVI' siècle, De la antigua lengua Poblaciones y comarcas de las Españas et le contenu du premier de Larramendi Discurso histórico de la Famosa Cantabria. Le livre fut un prélude à la lettre que Garat écrivit à Napoléon $I^{\sigma}$ pour la réunion de sept provinces dans un royaume de la Nouvelle Phénicie.

Cet ensemble d'observations nous montre l'effervescence de la pensée dans un coin prétenduement isolé de l'Europe où l'Inquisition espagnole saisit les 2/3 des encyclopédies de la péninsule et qui au siècle précédent connut les débuts du jansénisme.

Comme dans tous ses écrits dans Sobre los fueros de Guipuzcoa, I'identité basque est définie par

un peuple qui $\mathrm{n}^{\circ}$ a pas connu le servage

un peuple qui possède "la noblesse générale"

un peuple qui choisit ses rois

un peuple qui fait ses lois

un peuple qui se défend mais n'attaque pas hors de ses limites

un peuple dont la langue est l'euskara

un peuple qui met les biens indivis au dessus des biens particuliers

un peuple prêt à se battre pour ses libertés.

A cela s'ajoutent des arguments obsolètes tels la supériorité des Basques en métallurgie parce qu'ils seraient les descendants du Tubal biblique, justifiant leur esprit d'indépendance par la prétention d'être les descendants des Cantabres qui résistèrent aux Romains, mythes historiques mal fondés, alors que nos ancêtres avaient su si bien repousser les dominations franques et wisigothiques pendant deux siecles et auraient pu en tirer gloire.

Certains des arguments de Larramendi seront repris par Sabino Arana Goiri, mais le jésuite au XVIII siècle avait vu plus clair que le Biscaien ; par contre Sabino, l'époque étant plus propice, créa un Parti Nationaliste Basque, qui diffusa son idéologie de manière plus efficace. 
L'originalité de l'analyse larramendienne réside dans la définition et le projet d'organisation d'un état basque, d'une identité politique euskarienne, thème sur lequel i] est le premier penseur, mais sa notion du nationalisme n'est pas celle plus stricte, économiquement mieux définie, des $\mathrm{XIX}^{e}$ et $\mathrm{XX}^{\mathrm{e}}$ siècles, c'est le fruit antérieur à la bourgeoise Révolution française de 1789. Cette idéologie n'est pas sans rappeler l'utopie que les jésuites réussirent à maintenir parmi les Indiens guaranis sur les confins de l’Argentine du Brésil et du Paraguay et que le frlm « Missions » de De Niro a tenté d'illustrer.

Par contre la réflexion du père jésuite sur l'histoire basque est encore imprégnée des théories de la Renaissance sur le tubalisme et le cantabrisme, malgré les judicieuses observations de l'historien Oihenart dans sa Notitia de 1638 ; enfin sa vision de la langue basque est presque totalement inspirée, par les écrits inédits en euskara antérieurs à son œuvre - du Docteur Etcheberri de Sare et qu'il reprend, parfois, mot pour mot. Ce demier vécut à Vergara au Guipuzcoa : en effet, nous savons que ses manuscrits du début du XVIII' siècle passèrent entre les mains de Larramendi.

Quant à l'idée que le basque s'était parlé dans toute l'Espagne elle provient des écrits du licencié biscaien Poza au XVI' siècle et de l'œuvre d'Esteban de Garibay, historien du roi d'Espagne à la même époque, idée répétée au cours des siècles malgré les doutes scientifiques émis, dans ce cas encore, par Oihenart au XVII siècle.

\author{
Txomin PEILLEN \\ UPRESA du CNRS \\ UPPA
}




\section{Bibliographie}

COLLECTIF (bilingue) sous la direction de J.A. LAKARRA. Manuel Larramendiren mendeurrena (1690-1990) Andoain, 1992

ETXEBERRI Joanes, Joanes Etxeberri Sarakoaren lan hautatuak Ed. Kriselu Lur Donostia, 1972 (manuscrit de 1715) Traité d'enseignement du latin et du basque.

EGIATEGI (Jüsef) Lehen libüria edo Filosofo Heüskaldünaren Ekeia (1785), Txomin Peillen, Ed. Euskaltzaindia, Bilbao, 1983.

LARRAMENDI (Manuel de) S.J.De la antigüedad y universalidad del Bascuence en España, Salamanca 1728

- El imposible vencido. Ane de la lengua vascongada, Madrid 1729 (grammaire)

- Discurso historico sobre la famosa Cantabria, Madrid, 1736.

- Diccionario trilingüe Castellano, Bascuence y latin, Donostia, 1745

- Conferencias curiosas politicas, legales y morales Sobre los fueros de la M.N. y M.L. Provincia de Guipuzcoa. (1745-1765) Publiées pour la première fois aux Ed. Edili Donostia, 1983.

OIHENART Arnaud Notitia utriusque Vascoiniae tum Ibericae tum Aquitanicae, Paris 1638.

PEILLEN Txomin, "Jüsef Egiategi Larramendiren zuberotar jarraitzailea" [Egiategi disciple souletin de Larramendi] in Egan, Donostia, mars I963.

"Jüsef Egiategi historien -philosophe de langue basque au XVIII" siècle, Bulletin du Musée Basque, 2‘ trimestre, Bayonne 1984.

"Euskal aberri ezagutza ideologia testu ta literaturaren bitartez" in Ernaroa, Deustu-Bilbao, 1985 [historique de la conscience nationale basque par les textes idéologiques et la littérature].

POZA (Licenciado Andres de) De la antigua Lengua Poblaciones y Comarcas de las Españas, Ed. Mares Bilbao, 1597. Ed. Castilla Madrid, 1959. 